\title{
Pipeline for the identification and classification of ion channels in parasitic flatworms
}

\author{
Bahiyah Nor ${ }^{1 \dagger}$, Neil D. Young ${ }^{1 *+}$, Pasi K. Korhonen ${ }^{1}$, Ross S. Hall ${ }^{1}$, Patrick Tan ${ }^{2,3}$, Andrew Lonie ${ }^{4}$ \\ and Robin B. Gasser ${ }^{1 *}$
}

\begin{abstract}
Background: Ion channels are well characterised in model organisms, principally because of the availability of functional genomic tools and datasets for these species. This contrasts the situation, for example, for parasites of humans and animals, whose genomic and biological uniqueness means that many genes and their products cannot be annotated. As ion channels are recognised as important drug targets in mammals, the accurate identification and classification of parasite channels could provide major prospects for defining unique targets for designing novel and specific anti-parasite therapies. Here, we established a reliable bioinformatic pipeline for the identification and classification of ion channels encoded in the genome of the cancer-causing liver fluke Opisthorchis viverrini, and extended its application to related flatworms affecting humans.
\end{abstract}

Methods: We built an ion channel identification + classification pipeline (called MuSICC), employing an optimised support vector machine (SVM) model and using the Kyoto Encyclopaedia of Genes and Genomes (KEGG) classification system. Ion channel proteins were first identified and grouped according to amino acid sequence similarity to classified ion channels and the presence and number of ion channel-like conserved and transmembrane domains. Predicted ion channels were then classified to sub-family using a SVM model, trained using ion channel features.

Results: Following an evaluation of this pipeline (MuSICC), which demonstrated a classification sensitivity of $95.2 \%$ and accuracy of $70.5 \%$ for known ion channels, we applied it to effectively identify and classify ion channels in selected parasitic flatworms.

Conclusions: MuSICC provides a practical and effective tool for the identification and classification of ion channels of parasitic flatworms, and should be applicable to a broad range of organisms that are evolutionarily distant from taxa whose ion channels are functionally characterised.

Keywords: Ion channels, Identification, Classification, Parasitic flatworms, Bioinformatic pipeline

\section{Background}

Ion channels are pore-forming transmembrane protein complexes, whose functions include generating electrical signals (action potentials) by regulating the flow of ions across the membranes of cells, gating ion flow across epithelial and secretory cells, and governing cell volume [1].

\footnotetext{
* Correspondence: nyoung@unimelb.edu.au; robinbg@unimelb.edu.au ${ }^{\dagger}$ Equal contributors

${ }^{1}$ Faculty of Veterinary and Agricultural Sciences, The University of Melbourne, Parkville, Victoria 3010, Australia

Full list of author information is available at the end of the article
}

These channels are categorised physiologically based on their gating mechanisms (voltage-gated or ligand-gated) and the types of ions that they transport (e.g., $\mathrm{Ca}^{2+}, \mathrm{Cl}^{-}, \mathrm{K}^{+}$ and $\left.\mathrm{Na}^{+}\right)[1,2]$. Given that they have essential and specific roles in a wide range of different cells and that the disruption or mutation of their functions often causes serious disease [3], ion channels are recognised as valuable targets for drugs for many non-infectious disorders of humans and animals $[4,5]$.

Ion channel repertoires of some ("model") organisms, such as Homo sapiens (human) and Caenorhabditis 
elegans (free-living roundworm), are relatively well defined, because of the availability of extensive genomic, proteomic, functional and other datasets as well as ion channel functional information for these species (e.g., [6-10]), but this is not the case for most other organisms whose biology, biochemistry and physiology are largely unknown and are divergent from well-characterised organisms, such as humans and C. elegans [11]. This is particularly the case for eukaryotic pathogens, such as flatworm parasites (phylum Platyhelminthes), which are evolutionarily distinct from "model" species and cause devastating diseases of major proportion in humans and animals around the world [12].

As the management of many socioeconomically important parasitic flatworm diseases is often inadequate or compromised due to the inefficacy of some anthelmintics or emerging resistance [13, 14], identifying and characterising ion channel repertoires in flatworms could define novel and selective drug targets, and might open up avenues to design safe drugs with essentially no adverse effect on the human or animal hosts. Clearly, the massive expansion of genomic and transcriptomic datasets for a range of important parasitic flatworms, such as Opisthorchis viverrini, Clonorchis sinensis (liver flukes), Schistosoma haematobium, Schistosoma japonicum, Schistosoma mansoni (blood flukes), Echinococcus granulosus, Echinococcus multilocularis and Taenia solium (see Table 1), provides enormous scope to investigate the repertoires of ion channels in such worms. However, given their substantial molecular genetic and evolutionary divergence from well-characterised organisms, the challenge now is to reliably predict or identify as well as classify these channels from available molecular datasets.

The availability of large genomic datasets and the development of new bioinformatic approaches now make it feasible to classify ion channels using amino acid sequence and/or protein structural similarities. Generic bioinformatic tools, such as BLAST [15], HMMER [16] and InterProScan [17], are commonly used for gene annotation [18-20]. Besides these generic tools, some studies [21-23] have delivered algorithms specifically to classify ion channels, and most of them employ machine-learning algorithms trained using ion channel protein sequence data from specialised protein databases, such as IUPHAR (International Union of Basic and Clinical Pharmacology), LIC (ligand-gated ion channel) and VKCDB (voltage-gated potassium channel) [24-26]. Most functionally annotated and curated ion channels in the UniProtKB/SwissProt database are from deuterostomes (e.g., vertebrates) and ecdysozoans (e.g. C. elegans and Drosophila melanogaster). The integration of these data and use of advanced bioinformatics should significantly enhance our ability to explore (identify and classify) ion channels in eukaryotes that are evolutionarily distant from taxa whose ion channels are functionally characterised. To this end, the aim of this study was to establish a bioinformatic pipelines for the reliable identification and classification of ion channels in parasitic flatworms affecting millions of people and animals worldwide (Table 1). Our main focus here was on the cancer-causing (carcinogenic) liver fluke O. viverrini [27], and we extended its application to related flukes as well as socioeconomically important tapeworm parasites $[28,29]$ (Table 1).

\section{Methods}

We constructed and assessed a bioinformatics pipeline, called multi-screening ion channel classifier (MuSICC), to identify and classify ion channels (Fig. 1). This pipeline, which uses three existing bioinformatic tools and a support vector machine (SVM), was trained using known ion channel sequences obtained from public databases. Known sequences were subjected to multiple screening processes before being used to build the SVM models. For training, four internal databases were constructed: (i) known ion channel sequences used to construct the pipeline; (ii) conserved domain profiles for ion

Table 1 Salient information on parasites chosen for the present study

\begin{tabular}{|c|c|c|c|}
\hline Class/Family & Species & Disease & Key references \\
\hline \multicolumn{4}{|l|}{ Trematoda } \\
\hline \multirow[t]{2}{*}{ Opisthorchiidae (liver fluke) } & Opisthorchis viverrini & Opisthorchiasis; cholangiocarcinoma & {$[27,34]$} \\
\hline & Clonorchis sinensis & Clonorchiasis; cholangiocarcinoma & {$[62,63]$} \\
\hline \multirow[t]{3}{*}{ Schistosomatidae (blood fluke) } & Schistosoma haematobium & $\begin{array}{l}\text { Urogenital schistosomiasis; squamous } \\
\text { cell carcinoma of the bladder }\end{array}$ & {$[64,65]$} \\
\hline & Schistosoma japonicum & Hepatointestinal schistosomiasis & {$[66,67]$} \\
\hline & Schistosoma mansoni & Hepatointestinal schistosomiasis & {$[68-70]$} \\
\hline \multicolumn{4}{|l|}{ Cestoda } \\
\hline \multirow[t]{3}{*}{ (Taeniidae) } & Echinococcus granulosus & Cystic echinococcosis or hydatidosis & {$[28,71]$} \\
\hline & Echinococcus multilocularis & Alveolar echinococcosis or hydatidosis & {$[28,71]$} \\
\hline & Taenia solium & Cysticercosis & {$[29,71]$} \\
\hline
\end{tabular}




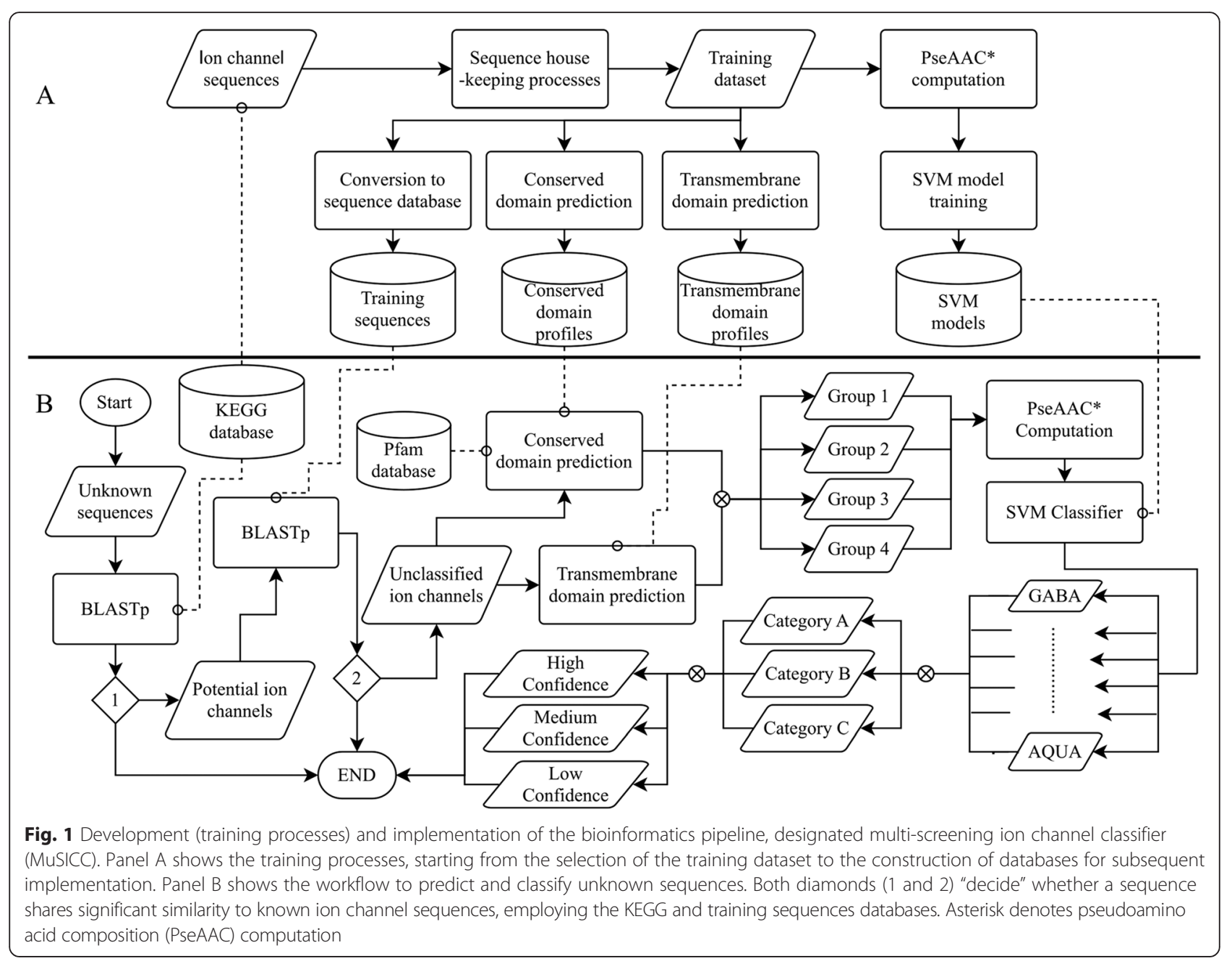

channels, (iii) transmembrane domain profiles for ion channels, and (iv) SVM models. The datasets as well as methods used for the prediction of ion channel proteins, and the construction and testing of SVMs are described in the following:

\section{Datasets}

Three datasets were prepared: (1) The training dataset was established using all classified ion channel and aquaporin sequence data from the KEGG database [30,31] as well as molluscan ion channel sequences in the UniProtKB/SwissProt Database [32], sodium channel protein 1 brain (Q05973; SCN1_HETBL), glutamate receptor (P26591; GLRK_LYMST), gamma-aminobutyric acid receptor subunit beta (P26714; GBRB_LYMST) and FMRFamideactivated amiloride-sensitive sodium channel (Q25011; FANA_HELAS). All UniProt/Swiss-Prot sequences were annotated using the KEGG orthology ion channel K-term of the KEGG entry, with highest sequence similarity inferred using BLASTp [15]. All human and C. elegans sequences and any sequences with ambiguous amino acid residues ("X", "B" or "Z"), or that were annotated as "hypothetical" or "putative", were removed from the training dataset. The training dataset was divided into 48 ion channel subfamily classes and one aquaporin class. Sequence similarity bias was removed from each subfamily class by selecting representative protein sequences of particular groups with $>80 \%$ sequence similarity using the CD-HIT program [33]. (2) The test dataset was established using all predicted proteins available for proteomes of human and C. elegans in the KEGG database $[30,31]$. (3) The parasite dataset represented amino acid sequences translated from genes of O. viverrini [34] and related flatworms $\mathrm{Cl}$. sinensis (liver flukes), S. haematobium, S. japonicum, S. mansoni (blood flukes), E. granulosus, E. multilocularis and T. solium (tapeworms) (Table 1).

\section{Prediction of ion channel proteins}

For the test and parasite datasets, ion channels were predicted based on amino acid sequence similarity searches (Fig. 1). To remove any 'false-positives' from 
these datasets, we initially screened each sequence against the KEGG database using BLASTp [15] (E-value of $<10^{-15}$ ), retaining proteins with a best match to an annotated ion channel. For the test dataset, a sequence similarity match to a human or $C$. elegans sequence in the KEGG database was ignored. Then, the remaining test and parasite dataset proteins were compared (BLASTp, E-value $<10^{-45}$ ) against the training dataset, with sequences similar to training dataset proteins retained as putative ion channel proteins.

For all sequences in each dataset, we identified conserved domains using InterProScan v.5.7.48 [35] and the Pfam database [36]. We curated the Pfam conserved domain accession numbers for individual sequences in the training dataset, to create conserved (C-) domain profiles for individual ion channel subfamilies. These profiles were then used to characterise and group sequences in the test and parasite ion channel datasets, based on the presence or absence of C-domains. Then, we predicted transmembrane (TM-) domains in individual sequences using TMHMM v.2.0 [37] and curated the number of TM-domains predicted from each sequence in the training dataset for each ion channel subfamily. The range of predicted TM-domains for sequences classified in each subfamily was then used to characterise and group sequences in test and parasite ion channel datasets. Finally, we divided putative test and parasite ion channels into four distinct groups according to: sequence similarity to known ion channels, and presence of C-domain and TM-domain(s) (Group 1); similarity, and presence of $\mathrm{C}$-domain, but no TM-domain(s) (Group 2); similarity, and presence of TM-domain(s), but no C-domain (Group 3); similarity, but no C- or TM-domains (Group 4).

\section{Construction and testing of support vector machines (SVMs)}

For each sequence in each dataset, we constructed the pseudo-amino acid composition [38] with $\lambda=55$, weight $=0.7$ and using established hydrophobicity values [39], hydrophilicity values [40] and side chain mass values [41]. We also determined the 400 character, dipeptide composition of each sequence in the dataset. The dipeptide composition $[f(x, y)]$ of any combination of two amino acid residues represented as $x$ and $y$, for each sequence was computed as

$$
\begin{aligned}
& \operatorname{sign}\left(a_{i},, a_{i+1}\right)=\left\{\begin{array}{l}
0 \text { if } a_{i}, a_{i+1} \neq x y \\
1 \text { if } a_{i}, a_{i+1}=x y
\end{array}\right. \\
& f(x, y)=\frac{\sum_{i=1}^{n-1} \operatorname{sign}\left(a_{i}, a_{i+1}\right)}{n-1}
\end{aligned}
$$

where $n$ is the length of the sequence and $a_{i}$ represents amino acid residue at position $i$. In total, each sequence was represented as a vector of 475 features, including the amino acid composition (20 characters), Chou's pseudo-amino acid composition $(\lambda=55)$ and dipeptide frequency (400 characters).

The SVMs were constructed using LIBSVM [42] extension in R v.3.2.0 [43] using the e1071 package [44]. For comparative purposes, five models were constructed using radial basis kernel, each with different sets of features and kernel parameters that were tuned with fivefold cross validation. The first model, named 'Amino', was built using 20 amino acid frequencies as features; the second model, called 'Chemistry', was built using 55 features based on the hydrophobicity, hydrophilicity and side chain-mass. The third model, 'Chou', was built using Chou's pseudo-amino acid composition by combining the 20 amino acid and 55 chemical information features. The fourth model, named 'Dipeptide', was built using 400 dipeptide composition features. The last model, 'Classifier,' was built using all 475 features.

The classification models were validated using five-fold cross-validation, and assessed against the classifications of known ion channel and aquaporin sequences encoded in the human and C. elegans genomes. Receiver operating characteristic (ROC) analysis [45] was conducted to evaluate the performance of each model. For comparative purposes, we also assessed the test dataset using other probabilistic classification methods, including random forest, classification via logistic regression and prior classifier, conducted using established methods [46-48]. Using the best-performing classification models, confusion matrices were constructed to further evaluate each model and compare their performance based on the final table of confusion. For the final model, the average classification probability values for individual subfamilies in the test dataset were computed; these probability values were utilised to classify the ion channels predicted from the parasite dataset.

Protein categories were classified based on SVM probability values: Category $A$ proteins had probability values greater than or equal to the subfamily probability threshold. Category $B$ proteins had probability values between $50 \%$ of the subfamily probability threshold and the subfamily probability threshold. Category $C$ proteins had probability values less than $50 \%$ of the subfamily probability threshold. A confidence ranking was given to our ion channel classifications. High confidence classifications included channels in Category A (Groups 1 to 4) and Category B (Groups 1 and 2), which were annotated by SVM subfamily classification. Medium confidence classifications included channels in Category B (Groups 3 and 4), which were annotated by SVM subfamilyclassifications and designated with the suffix, "-like" (e.g. GABA-like ion channel). Low confidence classifications included all proteins in Category $C$ (Groups 1 to 4), 
which represented ion channel-like proteins but could not be confidently assigned to a particular family or subfamily.

\section{Results}

\section{Training and test datasets}

The training dataset consisted of 26,050 classified ion channel and aquaporin sequences (Additional file 1: Table S1). After removing protein sequences from human and $C$. elegans as well as ambiguous sequences and sequence similarity bias from the dataset, 6299 classified ion channel and aquaporin sequences remained for model construction and training (Additional file 1: Tables S1 and S2). The test dataset consisted of the combined human and C. elegans proteins, including 389 sequences annotated with ion channel and aquaporin K-terms in the KEGG database (Additional file 1: Table S1).

\section{Identification of ion channels}

From the test dataset, 657 ion channel-like proteins with sequence similarity (BLASTp, E-value $<10^{-15}$ ) to known ion channels in the KEGG database were identified (Additional file 1: Table S3); they included 390 and 267 from humans and C. elegans, respectively, of which 299 human (100\%) and 93 C. elegans (100\%) ion channels were retained. Using a stringent sequence similarity search (BLASTp, E-value $<10^{-45}$ ) against sequences in the training dataset, 344 human and 185 C. elegans sequences were retained (Additional file 1: Table S3), including 299 human (100\%) and 93 C. elegans (100\%) ion channels.

A total of 194 unique Pfam C-domains were detected in 6161 sequences $(\sim 97.8 \%)$ of the training dataset, with 88 unique C-domains detected in $>75 \%$ of the sequences of 45 ion channel subfamilies (Additional file 2: Figure S1), such as the neurotransmitter-gated ion channel ligand-binding domain (PF02931) in $>88 \%$ of the Cys-loop subfamilies. TM-domains were detected in $5774(\sim 91.7 \%)$ sequences in the training set, with the number of such domains varying from 1 to 22 per protein (Additional file 2: Figure S2), being within the expected range for individual ion channel subfamilies. TMs were not detected in 525 sequences (Additional file 2: Figure S2). Based on sequence similarity, and the presence/absence of conserved and TM-domains, the sequences from the test dataset were divided into Group 1 ( $n=443$; including 335 known ion channels), Group 2 (57; 44 known ion channels), Group 3 (15; 5 known ion channels) and Group 4 (14; 5 known ion channels). Sequences within individual groups were then subjected to ion channel classification (Additional file 1: Table S4).

\section{Ion channel classifiers}

The performance of each of the five SVM models to classify ion channels was assessed using the training dataset. For this purpose, any known non-ion channel sequences were removed. Based on the five-fold cross-validation, training and test accuracies (Additional file 1: Table S5), we concluded that the 'Dipeptide' (94.6\% test accuracy) and 'Classifier' (95.9 \% test accuracy) models out-performed the other three models (Additional file 1: Table S5). Confusion matrices for the 'Classifier' and 'Dipeptide' models were constructed to further evaluate the models, and to compare their performances based on the final table of confusion (Additional file 1: Table S6); the 'Classifier' model recorded the best overall scores (Additional file 1: Table S6).

The performance of the 'Classifier' model was evaluated using the complete test dataset (including protein sequences that were not ion-channels) and recorded a sensitivity of $95.2 \%$, an accuracy of $70.5 \%$ and a specificity of $0 \%$; this result was expected, as an SVM model had not been trained for protein sequences other than ion channels (i.e. "non-ion channel" sequences). This finding shows the importance of identifying ion channels prior to classifying them.

The performance of the SVM classifier and the other probabilistic classification methods (random forest, classification via logistic regression and prior classifier) were then compared using the test dataset, employing the sorted probability values to construct ROC curves for each classifier (Additional file 2: Figure S3). The areaunder-the-curve (AUC) for the SVM 'Classifier' was 0.911 , random forest classification was 0.9105 , the logistic regression classifier was 0.8211 and the AUC for prior classifier was 0.6701 . The SVM and random forest classifiers performed similarly, but due to the high dimensionality of the data, classification via SVM was preferred.

Overall, there was a correlation between the probability values for the test dataset and correctness of their classification (Additional file 2: Figure S4A). In general, classifications with probability values of $\geq 0.54$ tended to be correct, whereas those with lower probability values tended to be incorrect. When probability values were compared among ion channel subfamilies (Additional file 2: Figure $\mathrm{S} 4 \mathrm{~B}$ ), the average probability values for each subfamily ranged from $\sim 0.15$ to 0.91 (Additional file 2: Figure S4B). Based on these findings, we elected to infer confidence in future classifications made using the SVM classifier employing the average probability values for individual subfamilies (Additional file 2: Figure S4B), instead of using single threshold probability value for all ion channel classifications (Additional file 2: Figure S4A). Using the test dataset, we observed higher probability values for proteins identified as Group 1 and Group 2 ion channels (Additional file 2: Figure S5). The majority of ion channels in Groups 3 and 4 had classifier probability values of $<0.5$ (Additional file 2: Figure S5). 
Ion channels of Opisthorchis viverrini and other flatworms Using our MuSICC pipeline (Fig. 1b; available for download at https:/github.com/vetscience/ion-channel-classifier), a total of 114 ion channels were predicted (in $53 \mathrm{~h}$ using an Intel ES-2695 $2.4 \mathrm{GHz}$ processor with eight cores) from the draft genome of $O$. viverrini by BLASTp against the KEGG database and the training dataset, and identified the presence/absence of $\mathrm{C}$ - and TM-domains. Thereafter, these sequences were divided (in $3 \mathrm{~min}$ ) into the following groups: 84 sequences shared sequence similarity to known ion channels and contained ion channel C- and TM-domains (Group 1); 18 sequences with sequence similarity to an ion channel and expected C-domains, but lacked the expected TM-domain profile (Group 2); six sequences were similar to known ion channels contained an expected TMdomain profile but lacked the expected C-domains
(Group 3) and six sequences shared similarity to a known ion channel but did not contain expected Cand TM-domains (Group 4) (Fig. 2 and Additional file 1: Table S7).

These 114 predicted ion channels were classified using the established SVM classifier and average probability value thresholds for individual ion channel subfamilies (Fig. 2 and Table 2), 38 of which were classified, with high confidence, and three as sub-family-like ion channels proteins with medium confidence; and 73 were classified as ion channel-like proteins (with no family or sub-family assignment); 30 of 38 sequences classified with high confidence were grouped in Group 1, and eight were in Group 2. One of the sequences classified with medium confidence was in Group 3 and the other two were in Group 4. Of the 73 sequences classified with low confidence, 54 were in Group 1, 10 in Group 2, five in

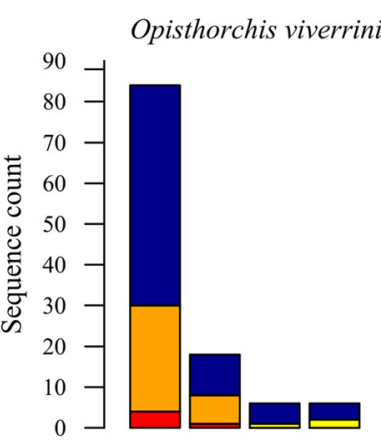

Schistosoma haematobium
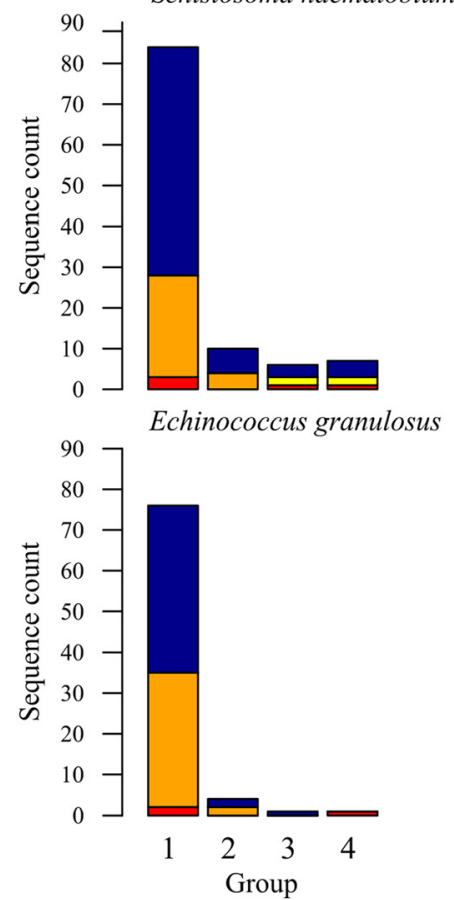

Clonorchis sinensis

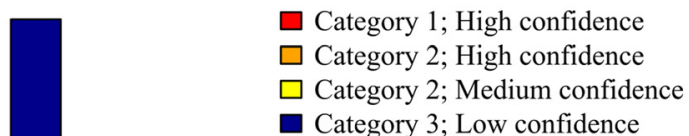

Schistosoma mansoni

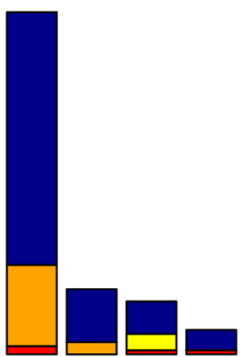

Echinococcus multilocularis

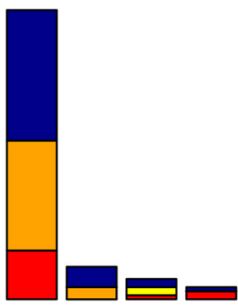

Taenia solium
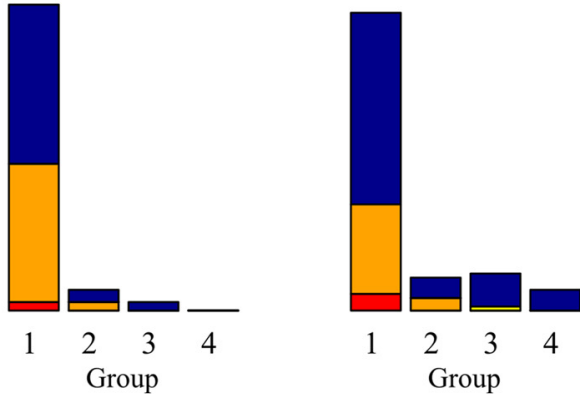

Fig. 2 Confidence in ion channel classifications for selected parasitic flatworms by group and classification category 
Table 2 Comparison of the numbers of ion channel sequences within each family among humans, C. elegans and representative parasitic flatworms ${ }^{*}$ that were classified with high and medium confidence

\begin{tabular}{|c|c|c|c|c|c|c|c|c|c|c|}
\hline \multirow[t]{2}{*}{ Ion channel family } & \multirow[t]{2}{*}{ Hs } & \multirow[t]{2}{*}{$\mathrm{Ce}$} & \multicolumn{5}{|c|}{ Trematoda } & \multicolumn{3}{|c|}{ Cestoda } \\
\hline & & & Ov & Cs & Sh & Sj & $S m$ & Eg & $E m$ & Ts \\
\hline Cys-loop superfamily & 46 & 36 & 3 & 2 & 4 & 5 & 4 & 6 & 6 & 7 \\
\hline Glutamate-gated cation channels & 18 & 8 & 4 & 6 & 6 & 0 & 4 & 5 & 5 & 5 \\
\hline Epithelial and related channels & 16 & 0 & 3 & 2 & 1 & 3 & 4 & 2 & 2 & 3 \\
\hline Ryanodine and IP3 receptors & 6 & 2 & 1 & 1 & 1 & 1 & 2 & 1 & 1 & 0 \\
\hline Voltage-gated ion channels & 114 & 22 & 16 & 15 & 14 & 11 & 16 & 12 & 13 & 7 \\
\hline Related to voltage-gated ion channels & 66 & 14 & 10 & 13 & 9 & 6 & 12 & 9 & 8 & 7 \\
\hline Chloride channels & 19 & 7 & 2 & 2 & 2 & 3 & 3 & 3 & 3 & 1 \\
\hline Aquaporins & 14 & 4 & 2 & 3 & 1 & 2 & 2 & 0 & 0 & 0 \\
\hline Unclassified ion channel-like proteins & - & - & 73 & 54 & 69 & 88 & 40 & 38 & 44 & 65 \\
\hline Total & 299 & 93 & 114 & 98 & 107 & 119 & 87 & 82 & 82 & 95 \\
\hline
\end{tabular}

*Hs Homo sapiens, Ce Caenorhabditis elegans, Ov Opisthorchis viverrini, Cs Clonorchis sinensis, Sh Schistosoma haematobium, Sj Schistosoma japonicum, Sm Schistosoma mansoni, Eg Echinococcus granulosus, Em Echinococcus multilocularis, Ts Taenia solium

Group 3 and four in Group 4 (Fig. 2). Then, the classification of ion channel sequences of $O$. viverrini was compared with those from human and C. elegans (Table 2). There were notable differences in the numbers of sequences for individual ion channel families between protostomes (O. viverrini and C. elegans). For O. viverrini, we classified eight calcium ion channels; five voltage-gated calcium ion channels; a ryanodine receptor, three CatSper, and two-pore channels. Therefore, O. viverrini was shown to have slightly more calcium ion channels $(n=8)$ compared with $C$. elegans $(n=6)$. The classification of $O$. viverrini ion channels showed a considerably higher number of sequences $(n=3)$ representing the "Epithelial and Related Channels" ion channel family compared with C. elegans $(n=0)$. One of "Epithelial and Related Channels" family in $O$. viverrini sequences was classified as a acid-sensing ion channel (ASIC), and two sequences as ATP-gated cation channels (P2X).

Logically extending this work, ion channel sequences from other flatworms, including $\mathrm{Cl}$. sinensis (liver fluke), S. haematobium, S. japonicum, S. mansoni (blood flukes), E. granulosus, E. multilocularis and T. solium (tapeworms), were predicted and classified using the established pipeline (Figs. 2 and 3, Table 2 and Additional file 1: Tables S7 and S8). For liver flukes, there were no marked differences in the number of sequences classified in individual ion channel families between $O$. viverrini and $C$. sinensis, although more voltage-gated cation channels could be classified with high confidence for the former species $(n=16)$ than the latter $(n=15)$ (Fig. 3 and Additional file 1: Table S8). For blood flukes, the total number of classified ion channel sequences varied considerably among $S$. haematobium, S. japonicum and S. mansoni (Figs. 2 and 3, Table 2 and Additional file 1: Tables S7 and S8). The most notable difference was in the number of sequences classified as Isk potassium ion channel $\left(\mathrm{K}^{+}\right.$channel, KCNE, [Isk]), with S. japonicum and S. haematobium having eight and four, respectively and S. mansoni having none. Although glycine receptors were not classified for liver flukes, S. haematobium and S. mansoni both had two, S. japonicum had one. For tapeworms, there were three differences among the three species; (i) E. granulosus and E. multilocularis had three Ether-a-go-go potassium channels each, and T. solium had none. (ii) T. solium one ASIC, the other species had none. (iii) E. granulosus and E. multilocularis each had one Ryanodine receptor, and $T$. solium had none. Most ion channels were relatively conserved among trematodes, even though the numbers of genes classified in individual families varied. For instance, we predicted and classified the P2X receptor (not conserved with $C$. elegans) in all eight species. In contrast, comparisons showed that the glycine receptors were not conserved between trematodes and cestodes. While glycine receptor genes could be classified for blood flukes, none were classified for the other flatworms studied. We also noted that there are more CatSper and two-pore channel genes classified in trematodes (average of three per species) than in the tapeworms (one per species) (Table 2 and Additional file 1: Tables S7 and S8).

\section{Discussion}

Here, we constructed a practical bioinformatic pipeline, designated MuSICC, to both identify and classify known ion channel families/subfamilies by combining three existing tools and an SVM classifier [49] trained using classified ion channel amino acid compositions, Chou's pseudo-amino acid compositions [38] and dipeptide frequencies. Although previous tools were developed to identify select ion channel groups [21, 23, 50, 51], none of them both identify and classify (all) ion channels into 


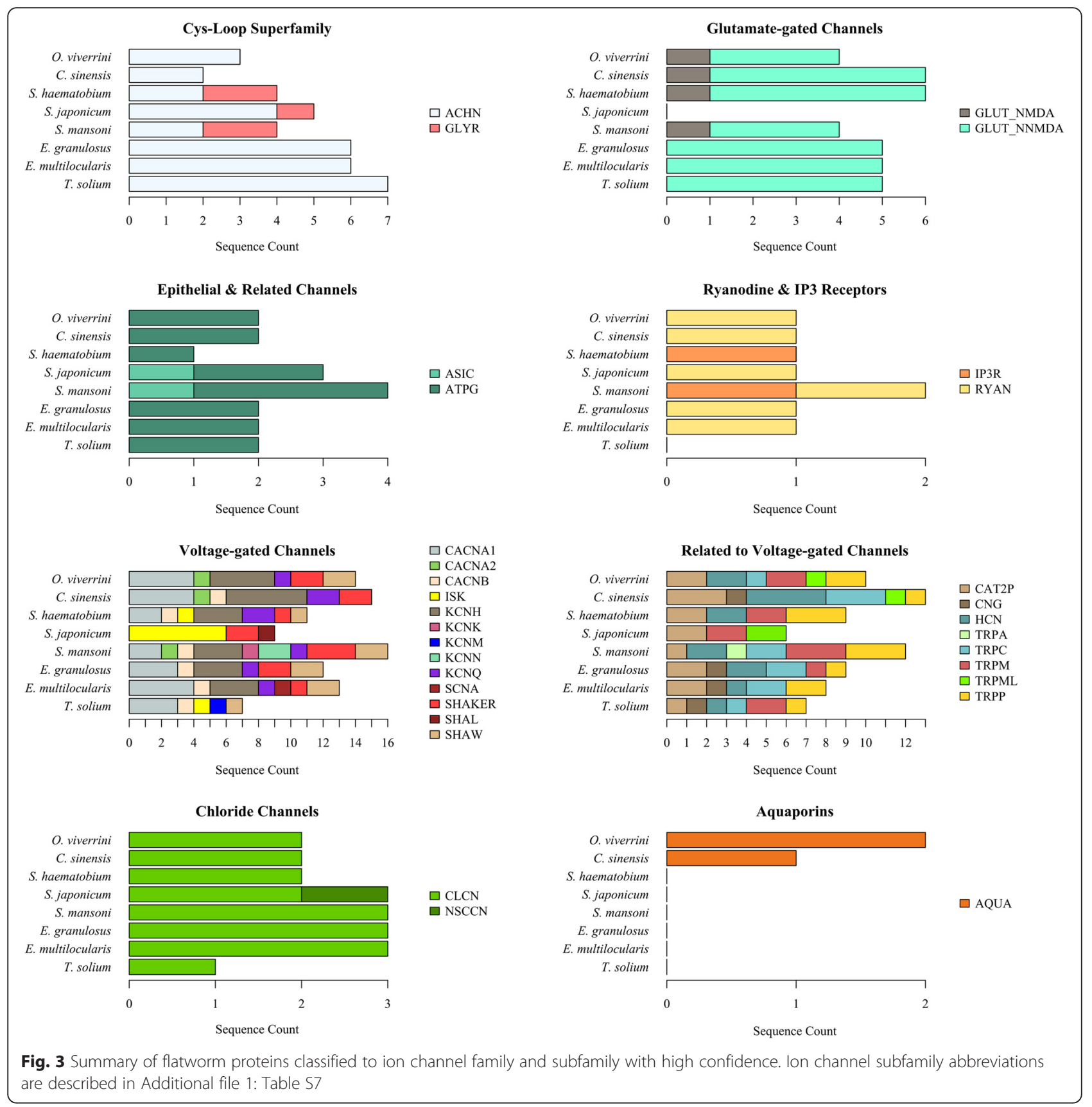

families and subfamilies. Here, we focused on developing a pipeline that would identify and classify such ion channels from eukaryotic organisms that are genetically and biologically very distinct from "model" organisms (such as $C$. elegans, Drosophila and humans, whose ion channels are well-characterised). The phylogenetic positions of parasitic flatworms in the eukaryotic evolutionary tree [52] made them ideal candidates for this study. Moreover, evidence that some flatworms are developing resistance against some of the recommended chemotherapies [13, 14] necessitates the search for new anthelmintics, and ion channels represent promising targets for such drugs $[4,5]$.
In this study, we first constructed and evaluated the pipeline to identify and classify channels in $O$. viverrini, a highly significant carcinogenic parasite affecting $>8$ million people worldwide [27]. Following this evaluation, we then applied this pipeline to datasets for seven other socioeconomically important flatworms (Table 1), and undertook a detailed, comparative analysis. The key to accurate identification and classification was the prediction process. As the SVM models were not trained using nonion channel sequences (i.e. there is non-ion channel classifier), these models are not able to distinguish between ion channel and non-ion channel sequences. Therefore, it is 
important that the prediction of ion channel sequences (data screening) is accurate. We defined three prediction criteria: (1) significant sequence similarity to known ion channels, (2) presence of ion channel C-domains, and (3) an appropriate number of TM-domains compared with known ion channels.

The sequence similarity (BLASTp) screening steps proved to be effective in filtering out the majority of non-ion channel sequences. In the test dataset, 137 sequences $(25.9 \%)$ were incorrectly identified as ion channels. We determined that 32 of the 137 'false-positives' did not encode ion channels but were very similar to the ion channel training sequences, whereas 105 sequences were not annotated using the KEGG database. We compared the annotations of these 105 sequences with those in the UniProtKB [53] and RefSeq [54] databases; 88 of the sequences were putative ion channels/proteins, and 17 were unknown/uncharacterised proteins. Therefore, we are confident that future predictions, based on the thresholds set here, will yield a low number of falsepositive results, if any at all.

Although conducting two BLASTp processes may be computationally exhaustive and somewhat time consuming, the same result was not achievable by conducting BLASTp only once against either KEGG database or the training sequences. Proteins that were not ion channels and shared high sequence similarity (BLASTp, E-value $<$ $10^{-45}$ ) with ion channels were first identified and excluded by initially screening against the complete KEGG database and selecting proteins with a match to an ion channel. An additional search of our curated training dataset ensured that false-positive results were minimised, and known ion channels were retained. As the accurate prediction of ion channels is the key to the performance of the present pipeline, we considered the computation time to be less of a priority, at this stage.

The application of three existing bioinformatics tools posed some limitations on the present pipeline. First, the pipeline is dependent on the KEGG database and the KEGG Orthology (KO) grouping method. KO grouping provided a hierarchical annotation based on K-terms, which eased the process of predicting ion channel sequences following the first BLASTp step. However, the implementation of the $\mathrm{KO}$ grouping method for predicting sequences was restricted to the annotated ion channel genes in the KEGG database. BLASTp analysis against protein databases without an established annotation system would make an automation process impossible, because manual annotation of ion channel sequences is not feasible as the number of sequences increases. An alternative to the $\mathrm{KO}$ annotation is the UniProt Gene Ontology Annotation (UniProt-GOA) database [55]. Second, the bioinformatic pipeline is dependent on the performance of the prediction tools applied - BLASTp, InterProScan and
TMHMM 2.0. Based on the present findings, the tools applied here allow the reliable prediction of ion channel sequences. However, the quality of sequences to be identified and classified needs to be high; the use of poor quality sequences will result in mis-classifications.

Two factors were considered crucial in relation to accepting or rejecting the classification made by the SVM classifier. The first was the probability value, computed by the classifier to determine the probability that an unknown sequence belonged to the classified ion channel subfamily, and enabling the probability thresholds to be defined for individual subfamilies (Additional file 2: Figure S4). The second factor considered was the groupings that were made based on the prediction criteria. There was a close association between grouping and the SVM classifier probability value (Additional file 2: Figure S5A); sequences classified with a probability of $>0.8$ were usually assigned to Groups 1 and 2 - the sequences with significant similarity to known ion channels and contained conserved domains of ion channels. Therefore, sequence grouping also provided confidence in the classification of ion channels.

Ion channels are of critical importance for the growth and development of flatworms [56] as well as neuromuscular function $[57,58]$. Ion channels can also play an important role in antiparasitic drug activity. For instance, calcium channels are thought to regulate praziquantel's disruption of $\mathrm{Ca}^{2+}$ homeostasis in adult worms [59] and nicotinic acetylcholine receptors (nAChRs) are targets of commercially available drugs that kill nematodes [60]. Most functionally characterised flatworm channels (Table 3) were identified and/or correctly classified using our bioinformatic pipeline (see Additional file 1: Table S8). Only four functionally characterised $S$. mansoni glutamate-gated chloride channel ( $\mathrm{SmGluCl}$ ) subunits [61] appeared to be misclassified using our pipeline, two (Smp_096480 and Smp_015630) of which were classified as glycine receptors in Category A/Group 1. In this instance, the accuracy of our classification is likely affected by a lack of conserved features or amino acid sequence between this novel flatworm clade of SmGluCl-like channels and functionally similar receptors in other eukaryotes [61]. Therefore, the under-representation of taxon-specific protein families in public sequence databases can affect the accuracy of the protein classifiers constructed using amino acid features; this observation emphasises the importance of a continual deposition of sequence data for non-model species into public databases. Furthermore, it is expected that improved draft genomes for parasitic flatworms are likely to enhance the predictions of ion channels and other genes. Despite the limitations of current 'omic resources for non-model species, our pipeline successfully classified a large proportion of the flatworm channels, many with high 
Table 3 lon channels of parasitic flatworms described in the published literature and whether they were identified and classified correctly using our bioinformatic pipeline (MuSICC)

\begin{tabular}{|c|c|c|c|c|c|}
\hline Name & Protein ID & Species & Identified/characterised ${ }^{*}$ & Classified & Key references \\
\hline Shaker-related $\mathrm{K}^{+}$channel & SKv1.1 & Schistosoma mansoni & $1 / 1$ & 1 & [72] \\
\hline $\mathrm{P} 2 \mathrm{X}$ receptor & $\operatorname{SchP} 2 X$ & Schistosoma mansoni & $3 / 3$ & 3 & [73] \\
\hline Nicotinic acetylcholine receptors & ShAR2beta & Schistosoma haematobium & $2 / 1$ & 2 & {$[60]$} \\
\hline $\mathrm{Ca}^{2+}$ channel beta subunits & - & Schistosoma mansoni & $1 / 1$ & 1 & {$[74,75]$} \\
\hline Novel glutamate-gated chloride channel subunits & SmGluCl & Schistosoma mansoni & $4 / 4$ & 0 & [61] \\
\hline Acetylcholine-gated chloride channels & SmACCs & Schistosoma mansoni & $3 / 2$ & 0 & [19] \\
\hline ATP-sensitive potassium channel & CsKir6.2 & Clonorchis sinensis & $0 / 1$ & 0 & {$[76]$} \\
\hline Aquaporins & OVAQP & Opisthorchis viverrini & $2 / 1$ & 2 & {$[77,78]$} \\
\hline
\end{tabular}

* Number of ion channels submitted to Swissprot (http://www.uniprot.org/) that are associated with the key references

confidence. These data were used to explore similarities and differences in ion channel subfamilies between flatworms and model organisms.

The number of calcium ion channels classified for $O$. viverrini was higher than for $C$. elegans and humans. The number of sequences encoding such channels in $O$. viverrini represents $\sim 19.5 \%$ of 41 sequences classified with confidence to encode ion channels. This is more than the proportion of calcium ion channels in H. sapiens ( $13.7 \%)$, and there was also considerable diversity compared with $C$. elegans and human. Although there are some channels $(\sim 40.8 \%)$ that are conserved among the three species, there are ion channels that are shared only by any two of these organisms. Notably, the acid-sensing ion channels (ASIC) and ATP-gated cation channels (P2X) present in both O. viverrini and human were absent from C. elegans.

The subsequent classification of ion channels from the seven other species of flatworms (trematodes and cestodes) further reinforced the genetic diversity between these parasites and the two well-characterised "model" organisms. The average probability values, which were lower than the thresholds computed by the SVM classifier, indicated that ion channels of these parasites are distinct from all presently known ion channels, despite being similar to them and containing the C-domains. Furthermore, more than half of the sequences were annotated as "unclassified ion channel-like proteins" based on the low probability values and the absence of ion channel C-domains. Importantly, the bioinformatics pipeline established here is able to identify and classify ion channels (with $95 \%$ accuracy), irrespective of sequence diversity. Nonetheless, it may be possible, in the future, to enhance the performance of the pipeline using structural similarity predictions and by training the SVM classifier using protein sequences other than ion channel to be able to distinguish ion channels from those that are not. However, this will require additional work as the process of selecting non-ion channel sequences, as the training dataset would need to include a substantial number of curated sequences from distinct groups of proteins from many different species of eukaryotes.

\section{Conclusions}

The present study delivers a practical and effective bioinformatic pipeline (MuSICC) for both the identification and classification of ion channels in parasitic flatworms of socioeconomic importance. MuSICC should be useful for the selection of high-priority candidates for functional genomic studies and for drug target discovery in parasitic flatworms. In addition, it might guide future investigations of the roles of ion channels in cellular processes and host-parasite interactions. Although applied to parasitic flatworms, the MuSICC pipeline should be applicable to classifying ion channels in a wide range of organisms.

\section{Additional files}

Additional file 1: Table S1. Sequence counts per ion channel family obtained from the KEGG and SwissProt databases and included in the training and test datasets. Table S2. Accession numbers of ion channels selected for support vector machine model training. Table S3. The number of sequences in the testing dataset before and after BLASTp analyses. Table S4. The number of identified test data sequences from humans and C. elegans within each group and divided into known ion channel and non-ion channel datasets. Table S5. Cross-validation, training and testing accuracies of each model. Table S6. Final tables of confusion matrices for the "Classifier" and "Dipeptide" models. Table S7. Summary of flatworm ion channels predicted using the MuSICC identification and classification pipeline with high and medium confidence. Table $\mathbf{S 8 .}$ Complete set of flatworm ion channels predicted using the MuSICC identification and classification pipeline. (XLS $2960 \mathrm{~kb}$ )

Additional file 2: Figure S1. The number of conserved domains common to $>75 \%$ of the sequences in each ion channel subfamily within the training dataset. The proportion of the sequences in the subfamilies that share the number of domains is given in the graph. The number of conserved domains is grouped according to the ion channel families. Figure $\mathbf{S 2}$. The range of transmembrane domains predicted in training dataset ion channel proteins. Range of transmembrane domains per subfamily, grouped according to each ion channel family. Figure S3. Receiver operating characteristic (ROC) curves for each probabilistic classification method. Figure S4. Probability values of each ion channel subfamilies computed during classification of sequences in the test dataset. Figure A shows the relation between the probability values and 
classifications made by SVM classifier. Figure B shows average probability values for individual ion channel subfamilies. The average values were grouped according to the ion channel families. Figure S5. Characteristics of putative ion channels identified and classified from the test dataset (human and C. elegans proteins). Panel A: Test sequences ordered by their SVM probability value, with their identification grouping presented on the second $y$-axis. Most of the sequences classified using high probability values were classified in Groups 1 and 2. Panel B: Confidence in test data ion channel classifications by group and classification category. (DOCX $1083 \mathrm{~kb}$ )

\section{Competing interests}

The authors declare that they have no competing interests.

\section{Authors' contributions}

Conceived and designed the study and supervised the project: NDY and RBG. Undertook the study and data analysis: BN, NDY and RBG. Contributed to analysis using various tools: PKK, RSH, PT and AL. Wrote the paper: BN, NDY and RBG. All authors read and approved the final version of the manuscript.

\section{Acknowledgements}

This project was also supported by a Victorian Life Sciences Computation Initiative (grant number VR0007) on its Peak Computing Facility at the University of Melbourne, an initiative of the Victorian Government (R.B.G. and A.L.). Funding from the Australian Research Council, the National Health and Medical Research Council (NHMRC) of Australia, Yourgene Bioscience and Melbourne Water Corporation is gratefully acknowledged (R.B.G. et al.). N.D.Y. holds an NHMRC Career Development Fellowship. P.K.K. is the recipient of a scholarship (STRAPA) from the University of Melbourne.

\section{Author details}

${ }^{1}$ Faculty of Veterinary and Agricultural Sciences, The University of Melbourne, Parkville, Victoria 3010, Australia. ${ }^{2}$ Genome Institute of Singapore, 60 Biopolis Street, Singapore 138672, Republic of Singapore. ${ }^{3}$ Cancer and Stem Cell Biology, Duke-NUS Graduate Medical School, Singapore 138672, Republic of Singapore. ${ }^{4}$ Victorian Life Sciences Computation Initiative (VLSCI), The University of Melbourne, Parkville, Victoria 3010, Australia.

\section{Received: 9 February 2016 Accepted: 5 March 2016}

\section{Published online: 16 March 2016}

\section{References}

1. Hillie B. Ion channels of excitable membrane. USA: Sinauer Associates; 2001.

2. Ackerman MJ, Clapham DE. Ion channels - basic science and clinical disease. N Engl J Med. 1997;336:1575-86.

3. Jentsch TJ, Hübner CA, Fuhrmann JC. Ion channels: Function unravelled by dysfunction. Nat Cell Biol. 2004;6:1039-47.

4. Jiang Z, Zhou Y. Using bioinformatics for drug target identification from the genome. Am J Pharmacogenomic. 2005;5:387-96.

5. Overington JP, Al-Lazikani B, Hopkins AL. How many drug targets are there? Nat Rev Drug Discov. 2006;5:993-96.

6. Coetzee WA, Amarillo Y, Chiu J, Chow A, Lau D, McCormack T, Moreno H, Nadal MS, Ozaita A, Pountney D, et al. Molecular diversity of $\mathrm{K}^{+}$channels. Ann N Y Acad Sci. 1999;868:233-55.

7. Conn PJ, Pin JP. Pharmacology and functions of metabotropic glutamate receptors. Annu Rev Pharmacol Toxicol. 1997;37:205-37.

8. Macdonald RL, Olsen RW. GABA receptor channels. Annu Rev Neurosci. 1994;17:569-602.

9. North RA. Molecular physiology of P2X receptors. Physiol Rev. 2002;82:1013-67.

10. Strange K. From genes to integrative physiology: Ion channel and transporter biology in Caenorhabditis elegans. Physiol Rev. 2003:83:377-415.

11. Riutort M, Álvarez-Presas M, Lazaro E, Sola E, Paps J. Evolutionary history of the tricladida and the platyhelminthes: an up-to-date phylogenetic and systematic account. Int J Dev Biol. 2012;56:5-17.

12. Welburn SC, Beange I, Ducrotoy MJ, Okello AL. The neglected zoonoses - the case for integrated control and advocacy. Clin Microbiol Infect. 2015;21:433-43.

13. Brennan GP, Fairweather I, Trudgett A, Hoey E, McCoy, McConville M, Meaney M, Robinson M, McFerran N, Ryan L, et al. Understanding triclabendazole resistance. Exp Mol Pathol. 2007:82:104-09.

14. Brockwell YM, Elliott TP, Anderson GR, Stanton R, Spithill TW, Sangster NC. Confirmation of Fasciola hepatica resistant to triclabendazole in naturally infected Australian beef and dairy cattle. Int J Parasitol Drugs Drug Resist. 2014;4:48-54.

15. Altschul SF, Gish W, Miller W, Myers EW, Lipman DJ. Basic local alignment tool. J Mol Biol. 1990;215:403-10.

16. Eddy SR. Profile hidden Markov models. Bioinformatics. 1998;14:755-63.

17. Zdonov EM, Apweiler R. InterProScan - an integration platform for the signature-recognition methods in InterPro. Bioinformatics. 2001;17:847-48.

18. Lee N, Chen J, Sun L, Wu SJ, Gray KR, Rich A, Huang MX, Lin JH, Feder JN, Janovitz EB, et al. Expression and characterization of human transient receptor potential melastatin 3 (hTRPM3). J Biol Chem. 2003;278:20890-97.

19. MacDonald K, Buxton S, Kimber MJ, Day TA, Robertson AP, Ribeiro P. Functional characterization of a novel family of acetylcholine-gated chloride channels in Schistosoma mansoni. PLoS Pathog. 2014;10:e1004181.

20. Scott JG, Warren WC, Beukeboom LW, Bopp D, Clark AG, Giers SD, Hediger M, Jones AK, Kasai S, Leichter CA, et al. Genome of the house fly, Musca domestica L. a global vector of diseases with adaptation to a septic environment. Genome Biol. 2014:15:466

21. Lin $H$, Ding $H$. Predicting ion channels and their types by the dipeptide mode of pseudo amino acid composition. J Theor Biol. 2011;269:64-9.

22. Liu W-X, Deng E-Z, Chen W, Lin H. Identifying the subfamilies of voltagegated potassium channel using feature selection technique. Int J Mol Sci. 2014;15:12940-51.

23. Saha S, Zack J, Singh B, Raghava GPS. VGlchan: Prediction and classification of voltage-gated ion channels. Geno Prot Bioinfo. 2006;4:253-58.

24. Donizelli M, Djite MA, Le Novere N. LGICdb: a manually curated sequence database after the genomes. Nucleic Acids Res. 2006;34:D267-D69.

25. Gallin WJ, Boutet PA. VKCDB: voltage-gated $\mathrm{K}^{+}$channel database updated and upgraded. Nucleic Acids Res. 2010;39:D362-D66.

26. Kenakin T. New concepts in pharmacological efficacy at 7TM receptors: IUPHAR Review 2. Br J Pharmacol. 2013;168:554-75.

27. Sripa B, Bethony JM, Sithithaworn P, Kaewkes S, Mairiang E, Loukas A, Mulvenna J, Laha T, Hotez PJ, Brindley PJ. Opithorchiasis and Opisthorchis-associated cholangiocarcinoma in Thailand and Laos. Acta Trop. 2011;120S:S158-S68.

28. Eckert J, Schantz PM, Gasser RB, Torgerson PR, Bessonov AS, Movsessian SO AT, Grimm F, Nikogossian MA. Chapter 4: Geographic distribution and prevalence. In: WHO / OIE Manual on Echinococcosis in Humans and Animals: a Public Health Problem of Global Concern. 2001.

29. Garcia HH, Moro PL, Schantz PM. Zoonotic helminth infections of humans: echinococcosis, cysticercosis and fascioliasis. Curr Opin Infect Dis. 2007;20:489-94.

30. Kanehisa M, Goto S. KEGG: Kyoto encyclopedia of genes and genomes. Nucleic Acids Res. 2000;28:27-30

31. Kanehisa M, Goto S, Sato Y, Kawashima M, Furumichi M, Tanabe M. Data, information, knowledge and principle: back to metabolism in KEGG. Nucleic Acids Res. 2014;42:D199-205.

32. The UniProt Consortium. UniProt: a hub for protein information. Nucleic Acids Res. 2014;43:D204-D12.

33. Li W, Godzik A. CD-HIT: A fast program for clustering and comparing large sets of proteins or nucleotide sequences. Bioinformatics. 2006;22:1658-59.

34. Young ND, Nagarajan N, Lin SJ, Korhonen PK, Jex AR, Hall RS, SafaviHemami H, Kaewking W, Bertrand D, Gao S, et al. The Opisthorchis viverrini genome provides insights into life in the bile duct. Nat Commun. 2014;5:4378.

35. Jones P, Binns D, Chang H-Y, Fraser M, Li W, McAnulla C, McWilliam H, Maslen J, Mitchell A, Nuka G, et al. InterProScan 5: genome-scale protein function classification. Bioinformatics. 2014;30:1236-40.

36. Finn RD, Bateman A, Clements J, Coggill P, Eberhardt RY, Eddy SR, Heger A, Hetherington K, Holm L, Mistry J, et al. The Pfam protein families database. Nucleic Acids Res. 2014;42:D222-D30.

37. Krogh A, Larsson B, von Heijne G, Sonnhammer ELL. Predicting transmembrane protein topology with a hidden Markov model: Application to complete genomes. J Mol Biol. 2001;305:567-80.

38. Chou KC. Prediction of protein cellular attributes using pseudo-amino acid composition. Proteins. 2001;43:246-55.

39. Tanford C. Contribution of hydrophobic interactions to the stability of the globular conformation of proteins. J Am Chem Soc. 1962;84:4240-47.

40. Hopp TP, Woods KR. Prediction of protein antigenic determinants from amino acid sequences. Proc Natl Acad Sci U S A. 1981;78:3824-28.

41. Shen HB, Chou KC. PseAAC: a flexible web-server for generating various kinds of protein pseudo amino acid composition. Anal Biochem. 2008;373:386-88.

42. Chang C-C, Lin C-J. LIBSVM: a library for support vector machines. ACM Trans Intell Syst Technol. 2011;2:1-27. 
43. R: A language and environment for statistical computing. R Foundation for Statistical Computing, Vienna, Austria. [http://www.R-project.org/]

44. e1071: Misc Functions of the Department of Statistics (e1071), TU Wien. R package version 1.6-4. [http://CRAN.R-project.org/package=e1071]

45. Pollack I, Decker LR. Confidence ratings, message reception, and the receiver operating characteristic. J Acoust Soc Am. 1958;30:286-92.

46. Anderson JA. In: Krishnaiah PR, Kanal LN, editors. Logistic discrimination. In Handbook of statistics, vol. 2. Amsterdam: North Holland; 1982. p. 169-91.

47. Breiman L. Random forests. Mach Learn. 2001;45:5-32.

48. Jain AK, Duin RPW, Mao J. Statistical pattern recognition: A review. IEEE Trans Pattern Anal Mach Intell. 2000;22:4-37.

49. Cortes C, Vapnik V. Support-vector networks. Mach Learn. 1995:20:273-97.

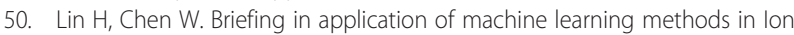
channel prediction. Sci World J. 2015;2015:945927.

51. Lin H, Li QZ. Using pseudo amino acid composition to predict protein structural class: Approached by incorporating 400 dipeptide components. J Comput Chem. 2007;28:1463-66.

52. Giribet G. Assembling the lophotrochozoan (=spiralian) tree of life. Phil Trans R Soc B. 2008;363:1513-22.

53. The UniProt Consortium. Activities at the Universal Protein Resource. Nucleic Acids Res. 2014:42:D191-D98.

54. Tatsutova T, Ciufo S, Fedorov B, O’Neill K, Tolstoy I. RefSeq microbial genomes database: new representation and annotation strategy. Nucleic Acids Res. 2014;42:D553-D59.

55. Huntley RP, Sawford T, Mutowo-Muellenet P, Shypitsyna A, Bonilla C, Martin MJ, O'Donovan C. The GOA database: Gene ontology annotation updates for 2015. Nucleic Acids Res. 2015:43:D1057-D10-63.

56. Ikeda T. Effects of blockers of $\mathrm{Ca}^{2+}$ channels and other ion channels on in vitro excystment on Paragonimus ohirai metacercariae induced by sodium cholate. Parasitol Res. 2004;94:329-31.

57. Greenberg RM. Ca ${ }^{2+}$ signalling, voltage-gated $\mathrm{Ca}^{2+}$ channels and praziquantel in flatworm neuromusculature. Parasitology. 2005;131:S97-S108.

58. Mendonca-Silva DL, Novozhilova E, Corbett PJR, Silva CLM, Noel F, Totten MIJ, Maule AG, Day TA. Role of calcium influx through voltage-operated calcium channels and of calcium mobilization in the physiology of Schistosoma mansoni muscle contractions. Parasitology. 2006;133:67-74.

59. Greenberg RM. Are $\mathrm{Ca}^{2+}$ channel targets of praziquantel action? Int J Parasitol. 2005;35:1-9.

60. Bentley GN, Jones AK, Agnew A. ShAR2beta, a divergent nicotinic acetylcholine receptor subunit from the blood fluke Schistosoma. Parasitology. 2007;134:833-40.

61. Dufour V, Beech RN, Wever C, Dent JA, Geary T. Molecular cloning and characterization of novel glutamate-gated chloride channel subunits from Schistosoma mansoni. PLoS Pathog. 2013;9:e1003586.

62. Huang Y, Chen W, Wang X, Liu H, Chen Y, Guo L, Luo F, Sun J, Mao Q, Liang $P$, et al. The carcinogenic liver fluke, Clonorchis sinensis: new assembly, reannotation and analysis of the genome and characterization of tissue transcriptomes. PLoS One. 2013;8:e54732.

63. Lun ZR, Gasser RB, Lai DH, Li AX, Zhu XQ, Yu XB, Fang YY. Clonorchiasis: a key foodborne zoonosis in China. Lancet Infect Dis. 2005;5:31-41.

64. Rollinson D. A wake up call for urinary schistosomiasis: reconciling research effort with public health importance. Parasitology. 2009;136:1593-610.

65. Young ND, Jex AR, Li B, Liu S, Yang L, Xiong Z, Li Y, Cantacessi C, Hall RS, $\mathrm{Xu}$ X, et al. Whole-genome sequence of Schistosoma haematobium. Nat Genet. 2012;44:221-5.

66. Liu F, Zhou Y, Wang ZQ, Lu G, Zheng H, Brindley PJ, McManus DP, Blair D, Zhang Q, Zhong $Y$ et al. The Schistosoma japonicum genome reveals features of host-parasite interplay. Nature. 2009;460:345-51.

67. McManus DP, Gray DJ, Li Y, Feng Z, Williams GM, Stewart D, Rey-Ladino J, Ross AG. Schistosomiasis in the People's Republic of China: the era of the Three Gorges Dam. Clin Microbiol Rev. 2010;23:442-66.

68. Berriman M, Hass BJ, LoVerde PT, Wilson RA, Dillon GP, Cerqueira GC, Mashiyama ST, Al-Lazikani B, Andrade LF, Ashton PD, et al. The genome of the blood fluke Schistosoma mansoni. Nature. 2009;460:352-58.

69. Colley DG, Bustinduy AL, Secor WE, King CH. Human schistosomiasis. Lancet. 2014;383:2253-64.

70. Protasio AV, Tsai IJ, Babbage A, Nichol S, Hunt M, Aslett MA, de Silva N, Velarde GS, Anderson TJC, Clark RC, et al. A systematically improved high quality genome and transcriptome of the human blood fluke Schistosoma mansoni. PLoS Negl Trop Dis. 2012;6:e1455.
71. Tsai IJ, Zarowiecki M, Holroyd N, Garciarrubio A, Sanchez-Flores A, Brooks KL, Tracey A, Robes RJ, Fragoso G, Sciutto E, et al. The genomes of four tapeworm species reveal adaptations to parasitism. Nature. 2013;496:57-63.

72. Kim E, Day TA, Bennett JL, Pax RA. Cloning and functional expression of a Shaker-related voltage-gated potassium channel gene from Schistosoma mansoni (Trematoda: Digenea). Parasitology. 1995;110(Pt 2):171-80.

73. Agboh KC, Webb TE, Evans RJ, Ennion SJ. Functional characterization of a P2X Receptor from Schistosoma mansoni. J Biol Chem. 2004;279:41650-57.

74. Salvador-Recatala V, Greenberg RM. The N terminus of a schistosome beta subunit regulates inactivation and current density of a $\mathrm{Ca}_{2}$ channel. J Biol Chem. 2010;285:35878-88.

75. Salvador-Recatala V, Schneider T, Greenberg RM. Atypical properties of a conventional calcium channel $\beta$ subunit from the platyhelminth Schistosoma mansoni. BMC Physiol. 2008;8:6.

76. Hwang SY, Han HJ, Kim SH, Park SG, Seog DH, Kim N, Han J, Chung JY, Kho WG. Cloning of a pore-forming subunit of ATP-sensitive potassium channel from Clonorchis sinensis. Korean J Parasitol. 2003;41:199-33.

77. Geadkaew A, von Bülow J, Beitz E, Tesana S, Grams SV, Grams R. Bi-functionality of Opisthorchis viverrini aquaporins. Biochimie. 2015;108:149-59.

78. Thanasuwan S, Piratae S, Brindley PJ, Loukas A, Kaewkes S, Laha T. Suppression of aquaporin, a mediator of water channel control in the carcinogenic liver fluke, Opisthorchis viverrini. Parasit Vectors. 2015:7:224.

\section{Submit your next manuscript to BioMed Central and we will help you at every step:}

- We accept pre-submission inquiries

- Our selector tool helps you to find the most relevant journal

- We provide round the clock customer support

- Convenient online submission

- Thorough peer review

- Inclusion in PubMed and all major indexing services

- Maximum visibility for your research

Submit your manuscript at www.biomedcentral.com/submit
BioMed Central 